Trauma Berufskrankh 2016 · [Suppl 3]:

18:S210-S213

DOI 10.1007/s10039-015-0095-4

Online publiziert: 24. November 2015

๑) Springer-Verlag Berlin Heidelberg 2015

CrossMark

Der Begriff Hygiene (hygieinos = gesund, heilsam) ist von der griechischen Göttin der Gesundheit abgeleitet. Hygieia war in der griechischen Mythologie die Tochter des Asklepios, des Gottes der Heilkunst. Diese enge Verbindung zwischen Hygiene und Heilkunst setzt sich fort bis in die Erkenntnisse unserer modernen Wissenschaft. Unser Wissen über die Ursachenzusammenhänge von Krankheiten und Erregern sind tatsächlich älter als die wissenschaftlichen Nachweise von Erregern selbst.

Im Jahr 1861 hat Ignaz Philipp Semmelweis seine bahnbrechenden Erkenntnisse zur Infektionsverhütung, die er über Jahrzehnte in gynäkologischen und geburtshilflichen Kliniken gesammelt hatte, unter dem Titel „Die Aetiologie, der Begriff und die Prophylaxe des Kindbettfiebers" veröffentlicht [1]. Dies geschah, obwohl die erste Sichtbarmachung und damit der erste Nachweis eines Bakteriums (Milzbranderreger) erst 1876 durch Robert Koch gelang [2,3].

Schon 30 Jahre zuvor (1847) hatte Semmelweis in seiner Klinik einen ersten Hygieneplan aufgestellt und diesen in einer Kohortenstudie evaluiert. Er ging aber noch von einer anderen Vorstellung über die Entstehung von Krankheiten und Infektionen aus. Erst mit dem mikrobiologischen Nachweis von Erregern, wie von Robert Koch praktiziert, wurden die Ideen von Semmelweis auch naturwissenschaftlich bestätigt. Es war dem Gynäkologen Semmelweis in seiner klinischen Tätigkeit aufgefallen, dass die in der Geburtshilfe verschiedenen Stationen zugeordneten Patientinnen unterschiedliche Komplikationsraten aufwiesen. Er konnte nachweisen, dass die Letalitätsrate in der geburtshilflichen Abteilung, in der Ärzte und Studenten arbeiteten, bei etwa 15-30\% lag, dagegen in der Abteilung, die

\author{
Michael Wich ${ }^{1,2} \cdot$ Axel Ekkernkamp ${ }^{1,3}$ \\ ${ }^{1}$ Unfallkrankenhaus Berlin, Berlin, Deutschland \\ ${ }^{2}$ Klinikum Dahme-Spreewald, Achenbach Krankenhaus, Königs Wusterhausen, Deutschland \\ ${ }^{3}$ Universitätsmedizin Greifswald, Greifswald, Deutschland
}

\section{Semmelweis und kein Ende}

von Hebammen geleitet wurde, nur bei $2 \%$.

Seine kritische Analyse dieser Beobachtung führte zu der Hypothese, dass die von Ärzten und Studenten vorgenommenen Leichensektionen zwischen den Entbindungen Ursache der erhöhten Erkrankungs- und Sterberate sein müssen. Er erklärte sich dies (noch in Unkenntnis der Existenz von Bakterien) mit einer weiteren Hypothese:

dass jedes Kindbettfieber, ..., durch die Resorbtion eines zersetzten thierischorganischen Stoffes entstehe.

Wir haben behauptet, dass dieser zersetzte thierisch-organische Stoff, welcher resorbirt das Kindbettfieber hervorbringt, in der Mehrzahl der Fälle den Individuen von aussen beigebracht werde, ... ein zersetzter Stoff den Individuen von
Der Träger, mittelst welchem am häufigsten
Aussen eingebracht wird, ist der untersuchende Finger.

Zum Beweis dieser Hypothesen schlussfolgerte er damals, dass eine Desinfektion der Hände nach einer Sektion und vor dem Patientenkontakt die Erkrankungsund Sterberate senken müsse. Durch das Aufstellen und konsequentes Umsetzen eines von ihm entwickelten Hygieneplanes erreichte er eine signifikante Senkung der Letalitätsrate auf $3 \%$.

Getragen durch diesen persönlichen Erfolg, suchte er konsequent nach weiteren Übertragungsmöglichkeiten und stellte als weitere Hypothese die folgende Überlegung auf: Auch andere „Verschmutzungen“ führen zu einer erhöhten Erkrankungsund Sterberate. Die Desinfektion der Hände, der Tücher und Instrumente vor jeder Untersuchung einer Schwangeren senkt die Erkrankungs- und Sterberate.

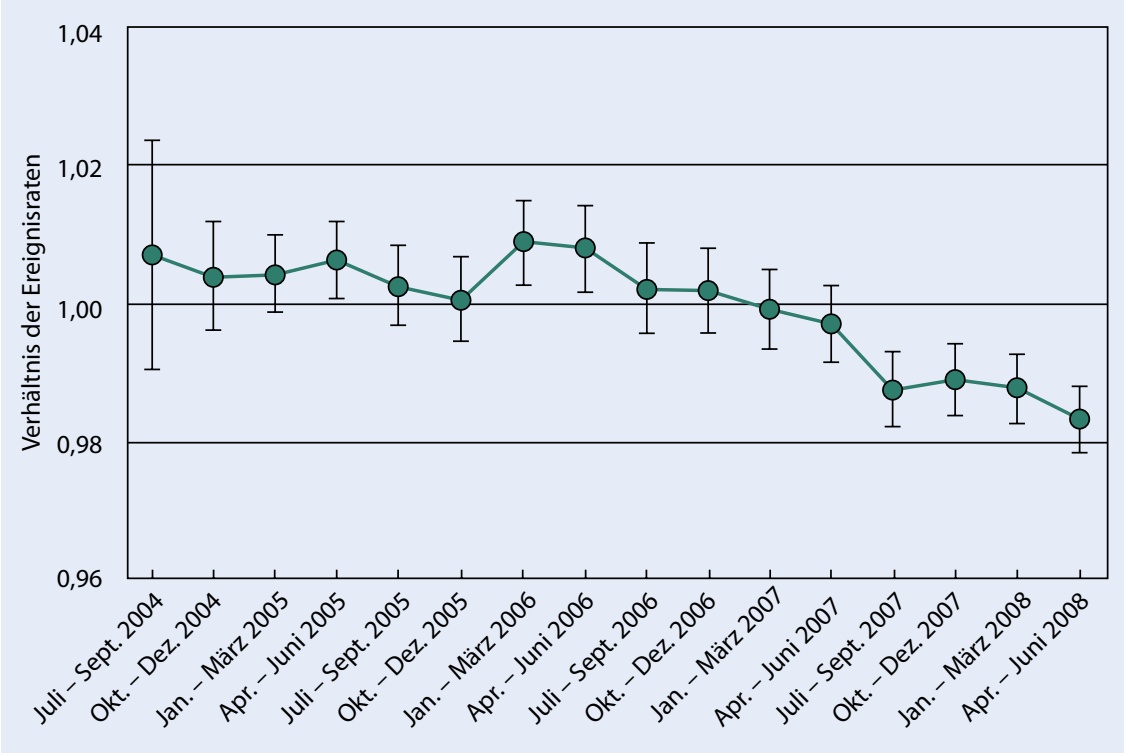

Abb. 1 \ Verhältnis der Ereignisraten zwischen $1 \mathrm{ml} / \mathrm{Tag} /$ Bett verbrauchtem alkoholischem Händedesinfektionsmittel und MRSA (multiresistenter Staphylococcus aureus)-Bakteriämie (95\%-Konfidenzintervall) 
Tab. 1 Anstieg des Händedesinfektionsmittelverbrauches (HDMV) von 2007 bis 2010 in

159 Krankenhäusern (AKTION Saubere Hände, 4. Erfahrungsaustausch)

\begin{tabular}{|c|c|c|c|c|c|c|}
\hline & & HDMV (ml/PT) & Differen & & Differen & 2007 \\
\hline & & & (ml/PT) & & (\%) & \\
\hline Jahr & Stationsart & Median & Median & p-Wert & Median & p-Wert \\
\hline 2007 & Alle & 18 & & & & \\
\hline 2008 & Alle & 19,8 & 2,1 & $<0,001$ & 10,5 & $<0,001$ \\
\hline 2009 & Alle & 23,8 & 5,4 & $<0,001$ & 29,8 & $<0,001$ \\
\hline 2010 & Alle & 25,1 & 6,8 & $<0,001$ & 35,9 & $<0,001$ \\
\hline 2007 & Intensiv & 65,8 & & & & \\
\hline 2008 & Intensiv & 72,3 & 6,6 & $<0,001$ & 12,8 & $<0,001$ \\
\hline 2009 & Intensiv & 84,7 & 14,2 & $<0,001$ & 32,7 & $<0,001$ \\
\hline 2010 & Intensiv & 88,9 & 17 & $<0,001$ & 40,9 & $<0,001$ \\
\hline 2007 & Normal & 14,7 & & & & \\
\hline 2008 & Normal & 16,4 & 1,9 & $<0,001$ & 10,6 & $<0,001$ \\
\hline 2009 & Normal & 19,7 & 4,9 & $<0,001$ & 22,3 & $<0,001$ \\
\hline 2010 & Normal & 21,2 & 6,3 & $<0,001$ & 27,7 & $<0,001$ \\
\hline
\end{tabular}

Tab. 2 Compliance-Beobachtungen im Rahmen der "Aktion Saubere Hände" - Referenz-

daten, Berechnungszeitraum 01.01.2014 bis 31.12.2014, erstellt am 27.05.2015

\begin{tabular}{|llllll|} 
Berufsgruppe & $\begin{array}{l}\text { Anzahl Kranken- } \\
\text { häuser }\end{array}$ & $\begin{array}{l}\text { Anzahl } \\
\text { Stationen }\end{array}$ & $\begin{array}{l}\text { Anzahl Be- } \\
\text { obachtungen }\end{array}$ & $\begin{array}{l}\text { Anzahl Hände- } \\
\text { desinfektionen }\end{array}$ & MW*1 \\
\hline Gesamt & & & & & \\
\hline Ärzte & 107 & 571 & 29.889 & 19.951 & $67 \%$ \\
\hline Pflege & 109 & 576 & 75.655 & 57.792 & $76 \%$ \\
\hline Sonstige & 101 & 506 & 15.265 & 9706 & $64 \%$ \\
\hline
\end{tabular}

$\mathrm{MW}^{*} 1$ gepoolter arithmetischer Mittelwert

Als Erfolg konnte er einen Rückgang der Sterberate auf 1,3\% (geringer als die der Hebammenstation) konstatieren.

Diese mehr als 150 Jahre alten Erkenntnisse passen sehr gut in die heutige epidemiologische Wissenschaft, die uns lehrt, dass Bevölkerungsalter und -gesundheit wesentlich mehr von der Ernährungssituation, den sozialen und hygienischen Verhältnissen abhängig sind als vom medizinischen Fortschritt. Rudolf Virchow, Mitbegründer der Zellularpathologie, hatte dies bereits während der 1848er-Revolution formuliert: „Medicin ist im Grunde eine sociale Wissenschaft und Politik ist nichts Anderes als Medicin im Großen. “ Wesentlich für Gesundheit sei „Freiheit mit deren Töchtern Bildung und Wohlstand“. Der medizinische Fortschritt steht seit Mitte des 19. Jahrhunderts wegen seiner zum Teil spektakulären Erfolge im Fokus der Mediziner und auch im Medieninteresse.

Die extrem wichtige Stellung der Hygiene gewinnt meist immer dann in der Öffentlichkeit an Bedeutung, wenn es sich um sog. Skandale bei der Missachtung von Hygienerichtlinien und -vorschriften handelt und wenn dadurch real oder vermeintlich Patienten zu Schaden kommen.

Hygiene im medizinischen Umfeld sollte aber nicht zum Schreckgespenst von medialen Horrorszenarien verkommen, sondern sie sollte als einfaches, sicheres und verlässliches Instrument einer Senkung von Komplikationsraten angesehen werden. Heute sind alle, die sich im Umfeld von medizinischen Einrichtungen bewegen, aufgefordert, Hygiene im Bewusstsein zu tragen und zu leben. Patienten, Pfleger, Ärzte, Reinigungskräfte, Besucher, Servicekräfte, Rettungsdienstmitarbeiter usw. sollten die Bedeutung elementarer Hygienevorschriften kennen und diese auch anwenden. Warum sollte eine Händedesinfektion, die heute auf Kreuzfahrtschiffen als Standard gilt, nicht auch genauso selbstverständlich im Kranken- haus oder in der Arztpraxis umsetzbar sein und von allen angenommen werden?

Bei den in immer wiederkehrender Regelmäßigkeit auftretenden Hygieneskandalen im Gesundheitsbereich geht es in der medialen Verarbeitung nach der Darstellung des Schadenszenarios schnell um eine Schuldzuweisung. Immer wieder zu hören sind die Argumente:

- Die Ärzte und die Pflegekräfte befolgen nicht die Händedesinfektionsregeln. In Sachen Hygiene herrschen in deutschen Kliniken Inkompetenz, Arroganz und Laisser-fair [4].

- Die Mitarbeiter in den Zentralsterilisationseinrichtungen sind schlecht ausgebildet und arbeiten schlampig [5].

- Die Krankenhausträger sparen zu viel Personal bei Ärzten und Pflegern ein, Hygienevorschriften können dadurch überhaupt nicht erfüllt werden. Es geht nur noch um Profitsteigerung, nicht mehr um den Patienten [6].

- Die Gesundheitspolitiker der Länder haben das Problem nicht erkannt und/oder nicht ernst genommen, entsprechende gesetzliche Vorgaben fehlen meist. Wie kann es sein, dass Hygiene im einen Bundesland anders geregelt ist als im Nachbarland? Schuld ist eine fehlende Bundesgesetzgebung [7].

Alle diese pauschalen Schuldzuweisungen helfen nicht, sie haben noch nie eine Infektion verhindert oder eine Komplikation vermieden. Alle im Gesundheitswesen Beschäftigten sollten sich auf die harten wissenschaftlichen Erkenntnisse beziehen, die uns heute zur Verfügung stehen. Der Zusammenhang zwischen dem Händedesinfektionsmittelverbrauch und der Reduktion der MRSA (multiresistenter Staphylococcus aureus)Sepsis-Rate ist mehrfach bewiesen.

In - Abb. 1 wird dargestellt, dass im Rahmen der vom britischen Gesundheitsministerium und der britischen National Patient Safety Agency initiierten Kampagne „cleanyourhands“ jeder mehr verbrauchte Milliliter Händedesinfektionsmittel (pro Tag/Bett) die MRSASepsis-Rate um ca. 1,0\% reduzieren konnte [8]. 
Ein Anstieg des Händedesinfektionsmittelverbrauches von $10 \mathrm{ml}$ pro/Tag/ Bett konnte in der genannten Studie das relative Infektionsrisiko um 9,6\% senken.

Auch in Deutschland hat die seit 2008 laufende „Aktion Saubere Hände“ ähnliche Erfolge in Krankenhäusern, in der ambulanten Medizin, in Alten- und Pflegeheimen vorzuweisen (• Tab. 1).

Dennoch sind wir hier von einem Idealzustand in der Umsetzung von Hygieneplänen und -richtlinien noch weit entfernt, wenn Ärzte in nur $67 \%$ aller notwendigen Situationen, in denen eine Händedesinfektion angezeigt wäre, diese auch durchführen (• Tab. 2).

Die zur Verbesserung dieser unbefriedigenden Situation notwendigen Maßnahmen sind auf allen Ebenen der medizinischen und pflegerischen Einrichtungen $\mathrm{zu}$ initiieren. Neben Schulungen und einer immer wiederkehrenden Erinnerung durch entsprechende Aktionen und Hinweise ist auch über ein Anreizsystem zu diskutieren, um die Compliance-Rate anzuheben. Die Händedesinfektion ist dabei aber nur ein - wenn auch extrem wichtiger - Parameter in der Vermeidung von Infektionen.

Ein weiterer Ansatzpunkt ist das Screening von Patienten auf Problemkeime [multiresistente Erreger (MRE)]. Problemkeime sind dabei Erreger, die eine Resistenz oder multiple Resistenzen auf Antibiotika aufweisen und damit extrem schwer angreifbar werden, wenn sie in einem Krankheitsgeschehen eine aktive Rolle spielen. In vielen Fällen sind die Patienten, die als Träger fungieren, nicht an einer entsprechenden Infektion erkrankt.

Der bekannteste Problemkeim, MRSA, der mit einer variablen und zum Teil hohen Kontaminationsrate einhergeht, hat mittlerweile erhebliche Konkurrenz unter den klinisch relevanten Resistenzbildnern bekommen. Es finden sich heute weltweit:

- VRE/GRE (Vancomycin- oder Glykopeptid-resistente Enterokokken),

- ESBL (Erweitertes-Spektrum- $\beta$-Lactamase produzierende Erreger: Escherichia coli, Klebsiellen u. a.) $[9,10]$

Trauma Berufskrankh 2016 · [Suppl 3]: 18:S210-S213 DOI 10.1007/s10039-015-0095-4 ๑) Springer-Verlag Berlin Heidelberg 2015

\section{Wich · A. Ekkernkamp}

\section{Semmelweis und kein Ende}

\section{Zusammenfassung}

Vor mehr als 150 Jahren hat Ignaz Philipp Semmelweis eine Methode zur Infektionsvermeidung entwickelt, die darin bestand, eine Händedesinfektion vor jedem Patientenkontakt durchzuführen. Diese - zunächst von den Fachkollegen abgelehnte - Methode hatte sich als effektiv erwiesen, um die Infektions- und Komplikationsrate seiner Patienten deutlich zu reduzieren. Erst 30 Jahre später wurden Bakterien als Infektionsursache identifiziert. Dadurch wurde der hypothetische Ansatz von Semmelweis auch wissenschaftlich unterstützt. In unserer heutigen Zeit kämpfen wir als Mediziner mit einer zunehmenden Zahl von Problemkeimen, die Resistenzen gegen eine Vielzahl von Antibiotika aufweisen. Die pharmakologische Entwicklung neuer Antibiotika kann damit nicht mehr Schritt halten.
Wir brauchen einen gewissenhafteren Umgang mit Antibiotika in der Massentierhaltung und der Humanmedizin, um den Selektionsdruck zu senken. WHO und auch der G7-Gipfel haben 2015 das Problem der zunehmenden Antibiotikaresistenzen in internationale Aktionspläne aufgenommen, um hier global effektiv gegenzusteuern. Semmelweis mit seinen Überlegungen der Infektionsvermeidung durch die Befolgung von Hygienevorschriften hat auch noch nach mehr als 150 Jahren ein wichtiges Instrument zur Infektionsbekämpfung vermacht.

\section{Schlüsselwörter}

Antibiotikaresistenzen - Problemkeime . Antibiotikaeinsatz · Händedesinfektion . Infektion

\section{Semmelweis and no end}

\section{Abstract}

More than 150 years ago Ignaz Philipp Semmelweis introduced a method of infection prevention involving disinfection of the hands, which could be carried out by every patient. This method proved to be an effective way to lower the rate of complications and infections in his patients. Some 30 years later bacteria were identified as the cause of infections and therefore provided scientific support for the hypothetical hygiene regulations inaugurated by Semmelweis. Nowadays physicians have to cope with an ever-increasing number of multidrug-resistant bacteria and the development of new antibiotics cannot keep up with the increasing number of resistant strains. A more rational appli- cation of antibiotics in animal factory farming and human medicine has to be accomplished in order to reduce the selection pressure. The World Health Organization (WHO) and the G7 summit meeting in 2015 have recognized the problem and both have announced global action plans for more rationale in treatment with antibiotics. Nevertheless, the regulations Semmesweis thought out 150 years ago to prevent infections are still the most powerful tools in the fight against infections.

\section{Keywords}

Antibiotic resistance - Problem pathogens . Antibiotic stewardship · Hand disinfection . Infection
- VRSA (Vancomycin-resistenter Staphylococcus aureus),

- KPC (Carbapenem-resistente Klebsiella pneumoniae),

- NDM-1 (New Delhi Metallo$\beta$-Lactamase 1, Escherichia coli, Klebsiella pneumoniae).

Eine Quelle der MRSA-Ausbreitung ist die Nutztierhaltung, die Nachweisraten von MRSA lagen zwischen 11,7 und 43,4\%. Untersucht wurden Kalb-, Schweine-, Hähnchen- und Putenfleisch. Die höchste MRSA-Nachweisrate be- traf Putenfleisch (2009: 43,4\%, 2010: $32,0 \%$, 2012: 37,7\%), gefolgt von Hähnchenfleisch (2009: 23,7\%, 2011: 27,7\%) [11]. In einer Untersuchung aus dem Jahr 2012 von Kola et al konnte in 399 Stichproben von deutschem, verkaufsfertigem Geflügelfleisch eine Rate von 43,9\% ESBL-bildenden Erregern nachgewiesen werden.

Die bei Nutztieren vorkommenden MRSA sind hauptsächlich dem klonalen Komplex CC398 zuzuordnen, in unterschiedlichem Ausmaß werden auch Isolate anderer klonaler Komplexe gefunden. 
Dies ist derzeit am häufigsten bei Geflügel der Fall, hier finden sich häufiger auch Isolate der klonalen Komplexe CC9 (Hähnchen) und CC5 (Pute). Diese Genotypen der Erreger wurden dann in Regionen mit hoher Tierdichte auch in etwa $10 \%$ der untersuchten Patienten mit MRSA wiedergefunden [12].

Das Screening von 18,7 Mio. Krankenhauspatienten pro Jahr auf alle Keime schafft einen Daten- und Kostenwust, der nicht zur Lösung des Problems beiträgt sondern neue schafft. Screening muss Sinn machen und Konsequenzen haben. Die Deutsche Krankenhausgesellschaft empfiehlt deshalb, ausgewählte Risikogruppen von Patienten zu testen. Dies sind neben Rückkehrern aus Risikoregionen auch Patienten, die aus anderen Kliniken oder Altenheimen eingewiesen werden [13].

Neben dem Screening spielt aber auch die Mitte 2009 eingeführte Meldepflicht für MRSA-Nachweise aus Blutkulturen oder Liquor ( $\$ 7$ Absatz 1 Satz 1 IfSG) eine entscheidende Rolle in der Eindämmung solcher Keime. Es sind gegenwärtig pro Jahr ca. 4000 Fälle in Deutschland, die den Gesundheitsämtern gemeldet werden [14].

Im engen Zusammenhang mit der Resistenzentwicklung und der Entstehung der oben genannten Problemkeine ist die Anwendung von Antibiotika in unserer Gesellschaft zu sehen. Zum einen steht die Nutztierhaltung seit etwa 10 Jahren im Fokus der Antibiotikaverabreichung und der damit einhergehenden Resistenzentwicklung. Seit etwa 2004 findet sich bei Schweinen in Deutschland und in den Nachbarländern nutztierassoziierter MRSA. In den letzten Jahren hat auch die Verbreitung von Darmbakterien in der Lebensmittelkette zugenommen, die gegen moderne Cephalosporine resistent sind. Beim Geflügel finden sich regelmäßig verschiedene Bakteriengruppen (z. B. Salmonella, Campylobacter, Escherichia coli und Staphylokokken), die gegen Fluorchinolone resistent sind. Im letzten Jahr wurden zudem erstmals gegen Carbapeneme resistente Keime bei Nutztieren in Deutschland nachgewiesen [15].

Aber auch Defizite im rationalen Antibiotikaeinsatz in der Humanmedizin haben den Keimselektionsdruck erhöht und die entsprechenden Resistenzen weiter verbreitet. Da sich dies in einer globalen Gesellschaft mittlerweile zu einem internationalen Problem ausgeweitet hat, wurde im Mai 2015 durch die WHO ein Aktionsplan zu Antibiotikaresistenzen [16] ins Leben gerufen. Das Thema Antibiotikaresistenzen findet sich auch in der Abschlusserklärung des G7-Gipfels vom 07. und 08.06.2015 auf Schloss Elmau [17]:

Antibiotika spielen für die heutigen und zukünftigen Erfolge in der Human- und Veterinärmedizin eine entscheidende Rolle. Wir unterstützen uneingeschränkt den kürzlich beschlossenen globalen Aktionsplan der WHO zu Antibiotikaresistenzen.

Eine wichtige Fortbildungsinitiative zum rationalen Umgang mit Antibiotika stellt das sog. Antibiotic-StewardshipProgramm dar. Hier schließt sich wieder der Kreis zu den Ideen von Ignaz Philipp Semmelweis von vor über 150 Jahren, denn auch im Antibiotic-StewardshipProgramm spielt die Zusammenarbeit mit der Hygiene, den „infection preventionists “ und den Epidemiologen eine wichtige Rolle.

\section{Korrespondenzadresse}

Prof. Dr. med. M. Wich
Klinikum Dahme-Spreewald,
$\begin{aligned} & \text { Achenbach Krankenhaus, } \\ & \text { Köpenicker Str. 29, 15711 } \\ & \text { Königs Wusterhausen } \\ & \text { m.wich@klinikum-ds.de }\end{aligned}$

\section{Einhaltung ethischer Richtlinien}

Interessenkonflikt. M. Wich und A. Ekkernkamp geben an, dass kein Interessenkonflikt besteht.

Dieser Beitrag beinhaltet keine Studien an Menschen oder Tieren.

The supplement containing this article is not sponsored by industry.

\section{Literatur}

1. Semmelweis IP (1861) Die Aetiologie, der Begriff und die Prophylaxe des Kindbettfiebers. C. A. Hartleben's Verlags-Expedition, Leipzig

2. Koch R (1876) Die Ätiologie der Milzbrand-Krankheit, begründet auf die Entwicklungsgeschichte des Bacillus Anthracis. J. A. Barth, Leipzig
3. Münch R (2003) Robert Koch und sein Nachlass in Berlin. De Gruyter Verlag, Berlin

4. http://www.tagesspiegel.de/berlin/krankenhaus-keime-wie-konnte-es-zu-den-infektionenkommen/7281406.html. Zugegriffen: 28. Sept. 2015

5. http://www.spiegel.de/wissenschaft/medizin/ tote-fruehchen-an-bremer-klinik-prueferentdeckten-schwere-fehler-a-870719.html. Zugegriffen: 28. Sept. 2015

6. http://www.morgenpost.de/printarchiv/berlin/ article106034847/Einige-Krankenhaeuser-wollendie-Hygiene-immer-noch-wegsparen.html. Zugegriffen: 28. Sept. 2015

7. http://www.zeit.de/politik/2011-01/krankenhaushygiene-keime. Zugegriffen: 28. Sept. 2015

8. Stone SP, Fuller C, Savage J et al (2012) Evaluation of the national Cleanyourhands campaign to reduce Staphylococcus aureus bacteraemia and Clostridium difficile infection in hospitals in England and Wales by improved hand hygiene: four year, prospective, ecological, interrupted time series study. BMJ 344:e3005

9. Eller $C$ et al (2013) Presence of b-lactamases in extended-spectrum-cephalosporinresistant Salmonella enterica of 30 different serovars in Germany 2005-11. J Antimicrob Chemother 68:1978-1981

10. Kola A et al (2012) High prevalence of extended-spectrum-b-lactamase-producing Enterobacteriaceae in organic and onventional retail chicken meat, Germany. J Antimicrob Chemother 67:2631-2634

11. http://www.bvl.bund.de/SharedDocs/Downloads/01_Lebensmittel/04_Zoonosen_ Monitoring/Zoonosen_Monitoring_Bericht_2013. pdf?_blob=publicationFile \&v=3. Zugegriffen: 28 . Sept. 2015

12. http://www.bfr.bund.de/de/fragen_und_ antworten_zu_methicillin_resistenten_ staphylococcus_aureus_mrsa_-11172. html\#topic_188342.Zugegriffen: 28. Sept. 2015

13. http://www.krankenhaushygiene.de/pdfdata/ task_force_mrsa_langfassung.pdf. Task force MRSA zur Intensivierung der Präventionsstrategien für die Eindämmung von MRSA Stellungnahme der DGKH. Zugegriffen: 28. Sept. 2015

14. Deutscher Bundestag Drucksache 18/3600 18. Wahlperiode 18.12.2014. Bericht der Bundesregierung über nosokomiale Infektionen und Erreger mit speziellen Resistenzen und Multiresistenzen http://www.dip21.bundestag.de/ dip21/btd/18/036/1803600.pdf. Zugegriffen 28. Sept. 2015

15. (2013) Antibiotikaresistenz in der Lebensmittelkette Tagungsband zum BfR-Symposium am 11. und 12. November 2013. Bundesinstitut für Risikobewertung, Berlin. http://www.bfr.bund.de/ $\mathrm{cm} / 350 /$ antibiotikaresistenz-in-der-lebensmittelkette-tagungsband.pdf. Zugegriffen 28. Sept. 2015

16. http://apps.who.int/gb/ebwha/pdf_files/WHA68/ A68_Jour8-en.pdf?ua=1. Sixty-eighth world health assembly A68/A/CONF./1 Rev.1 Agenda item 15.125 May 2015. Zugegriffen: 28. Sept. 2015 (Global action plan on antimicrobial resistance)

17. https://www.bundesregierung.de/Content/DE/ Anlagen/G8G20/2015-06-08-g7-abschluss-deu.pd $\mathrm{f}$ ?blob=publicationFile\&v=4. Abschlusserklärung G7-Gipfel 7.-8. Juni 2015, G7 Germany Schloss Elmau. Zugegriffen: 28. Sept. 2015 